\title{
THE INFLUENCE OF THE MICROWAVE POWER ON JOSEPHSON MICROWAVE ABSORPTION IN HIGH-TEMPERATURE SUPERCONDUCTORS
}

\author{
B. CzyżaK and J. Stankowski \\ Institute of Molecular Physics, Polish Academy of Sciences \\ Smoluchowskiego 17/19, Poznań, Poland \\ (Received July 26, 1990; in final form March 25, 1991)
}

\begin{abstract}
The influence of the microwave power on the internal Josephson Junction System (JJS) in granular high $T_{c}$ superconductors was investigated using EPR methodology. Josephson Microwave Absorption signal was used to monitor local temperature of the Josephson Junction System while the bulk sample temperature was measured by a thermocouple. The effect of the JJS overheating is discussed as the consequence of an interaction between JJS, microwave field and the bulk sample.
\end{abstract}

PACS numbers: $74.50 .+\mathrm{r}, 74.70 . \mathrm{Vy}$

\section{Introduction}

Shortly after the discovery of high-temperature superconductors by J.G. Bednorz and K.A. Mueller [1], intensive investigations of magnetic properties of these materials have begun. Many authors have observed low-field microwave absorption $[2-6,11-12,15-16]$ in these materials. This phenomenon was called Josephson Microwave Absorption (JMA) [2].

Microwave absorption has a complex nature; it is caused by the fluctuation of the electron wave function phase in zero magnetic field area (low-field peak) and by nucleation and viscosity of fluxon-flow in high magnetic field [11]. The EPR technique, which allows the measurement of microwave power dissipated by the system of internal Josephson junctions in low magnetic field, reveals a JMA line in external magnetic field given by:

$$
P_{\mathrm{JMA}}=P_{0}\left[1-\left[\frac{\sin \left(\pi \phi / \phi_{0}\right)}{\pi \phi / \phi_{0}}\right]^{2}\right],
$$


where $P_{0}=R I_{\mathrm{c}}^{2}(0)$ is the maximum power absorbed by the Josephson Junction System (JJS), $\phi_{0}$ is the flux quantum, $\phi$ is the magnetic flux linking JJS. JMA, which appears exactly at the critical temperature $\mathbf{T}_{c}$, was used to monitor the JJS temperature [8]. The interaction between the microwaves, JJS and the bulk sample results in the overheating of the Josephson Junction System. Because of poor heat conductivity of superconducting grains, thermally-isolated internal junctions created between the grains are heated up to temperature $T^{*}$ measured by the JMA signal. This local temperature $T^{*}$ is higher than the temperature $T$ of the whole sample measured by the thermocouple. The difference between both temperatures is proportional to the power $P$ of microwaves irradiating the junction:

$$
\Delta T=T^{*}-T=\frac{a P}{K^{\prime}}
$$

where $a$ is a measure of the microwave power absorbed by JJS and $K^{\prime}=K A / \Delta x$, where $\Delta x$ is a distance of the order of the junction's thickness, $A$ is the junction area and $K$ is the heat conductivity factor. When the sample is irradiated by the microwaves, we observe a shift of the temperature $T_{c}^{\text {eff }}$ (defined as the temperature at which the JMA signal appears or disappears). An analogous effect was observed in the investigations of granular classical superconductors, such as $\mathrm{Pb}$ and $\mathrm{Nb}$ $[9-13,14]$. The sample irradiation by microwaves was found to cause a splitting in the JMA line. This effect was also observed to be due to the overheating of the JJS.

\section{Experimental details and results}

Monophase $\mathrm{YBa}_{2} \mathrm{Cu}_{3} \mathrm{O}_{6.9}$ sample of high quality was investigated using an EPR SE/X RADIOPAN spectrometer. The sample was placed in a standard $\mathrm{TE}_{102}$ microwave resonator and cooled by vapours of liquid nitrogen. The bulk sample temperature was measured by $\mathrm{Cu}-\mathrm{Constantan}$ thermocouple glued directly to the sample. The spectrometer magnet was replaced by Helmholtz coils $[8,12]$ fed by a triangular current signal from a generator and amplifier, yielding magnetic field $B_{0}$ sweep of an amplitude of $\pm 50 \mathrm{G}$. Magnetic field of second modulation at $100 \mathrm{kHz}$ was also applied. The sample temperature was stabilized with an accurancy of $0.3 \mathrm{~K}$. All measurements were carried out at a very small $\left(10^{-3} \mathrm{~s}\right)$ time constant of the electronic receiver of the EPR spectrometer. The microwave absorption was studied at the field sweep rate of $10 \mathrm{G} / \mathrm{s}$, at which it had been known to reveal hysteresis in an external magnetic field [16]. The applied amplitude of $100 \mathrm{kHz}$ modulation was of a few Gauss. The microwave power was controlled by the spectrometer attenuator.

At a constant temperature, the derivative of the JMA signal $\mathrm{d} P / \mathrm{d} B$ was studied in the magnetic field $B$, for different microwave powers. At $77 \mathrm{~K}$ the amplitude of the JMA derivative $\mathrm{d} P / \mathrm{d} B$ increased with the microwave power increasing in the range of $20-50 \mathrm{~mW}$. For the power higher than $60 \mathrm{~mW}$ the signal decreased, and for $P=89 \mathrm{~mW}$ it completely disappeared (Fig. 1(a)). At $86.5 \mathrm{~K}$ (Fig. 1(b)) $\mathrm{d} P / \mathrm{d} B$ vs. $P$ dependence had a similar character, with the maximum 

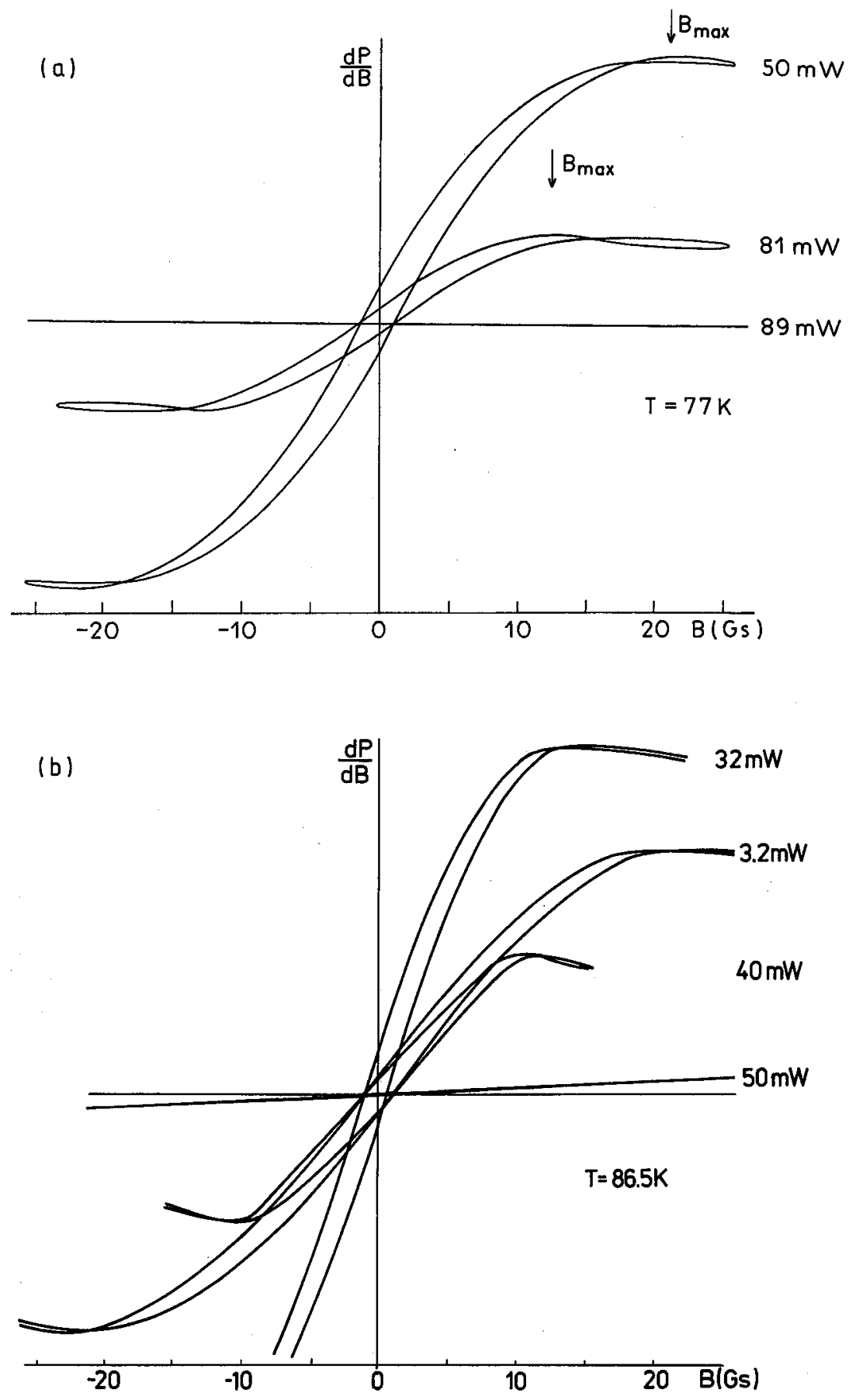

Fig. 1. The effect of microwave power on the microwave absorption at a constant temperature of bulk sample: $T=77 \mathrm{~K}(\mathrm{a}), T=86.5 \mathrm{~K}$ (b). 
for $P=25 \mathrm{~mW}$ and the signal disappeared at $50 \mathrm{~mW}$. During all measurements the temperature of the bulk sample measured by the thermocouple was constant. The characteristic feature is that the signal disappeared for the microwave power higher than $60 \mathrm{~mW}$. For $40 \mathrm{~mW}$, the signal was reproducible, clear, but after the increase in power to $60 \mathrm{~mW}$, it disappeared in $5 \mathrm{sec}$. Also, during the change of the microwave power from 60 to $40 \mathrm{~mW}$ - the signal arose in $5 \mathrm{sec}$. At $T=88.5$ $\mathrm{K}$ the amplitude of $\mathrm{d} P / \mathrm{d} B$ signal was observed to reach a maximum for $13 \mathrm{~mW}$ and disappear for $40 \mathrm{~mW}$. Of course, the amplitudes of the signal varied with temperature.

The influence of the microwave power on the $\mathrm{d} P / \mathrm{d} B$ maximum $B_{\max }$ was also studied. We suggest, $B_{\max }$ is proportional to lower critical field $B_{\mathrm{c} 1}$. The dependence of $B_{\max }$ on the microwave power irradiating the sample $P_{\text {micr }}$ can be described by the standard equation:

$$
B_{\mathrm{c} 1}\left(T, P_{\text {micr }}\right)=B_{\mathrm{c} 1}\left(0, P_{\text {micr }}\right)\left[1-\left(\frac{T^{*}}{T_{\mathrm{c}}}\right)^{2}\right],
$$

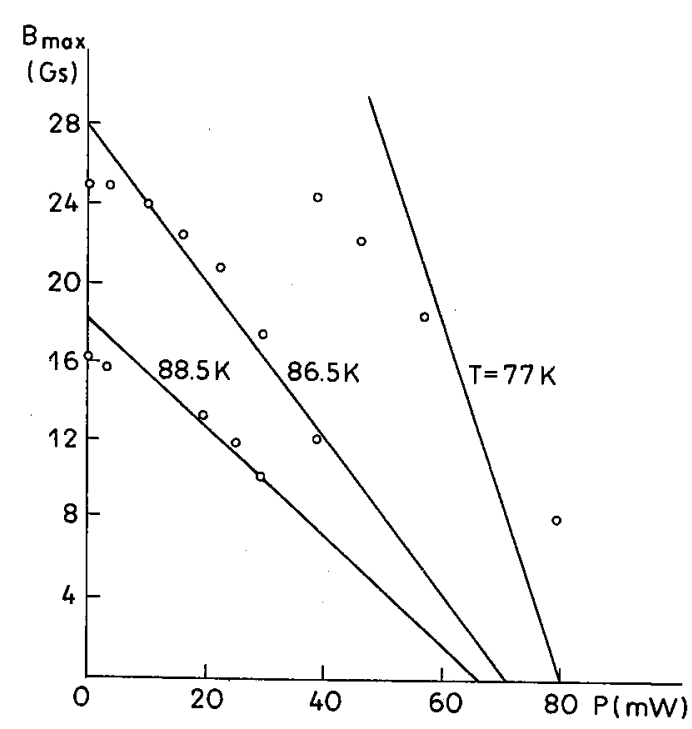

Fig. 2. The magnetic field $B_{\max }$ corresponding to the maximum of the $\mathrm{d} P / \mathrm{d} B$ signal vs. microwave power for different temperatures of bulk sample.

where $T^{*}$ is the local temperature of JJS; $T^{*}=T+\gamma P$. The coefficient $\gamma$ is inversely proportional to the heat conductivity factor. Close to $T_{\mathrm{c}}$ the value of the critical field $B_{\mathrm{c} 1}(0, P)=B_{\mathrm{cl} 1}(0,0)$ is independent of the microwave power. However (for $T<77 \mathrm{~K}$ ) $B_{\mathrm{c} 1}$ observed as $B_{\max }$ decreases with increasing amplitude of the oscillating microwave field. This effect has been reported already in [15]. Three lines decsribed by Eq. (3) for different temperatures are shown in Fig. 2. 
For $T_{\mathrm{c}}=92 \mathrm{~K}$ and temperatures $T_{1}=88.5 \mathrm{~K}, T_{2}=86.5 \mathrm{~K}, T_{3}=77 \mathrm{~K}$ we obtained a very good agreement with the experiment for $B_{\mathrm{c} 1}(0,0)=240 \mathrm{G}$ and for $\gamma_{1}=$ $0.052, \gamma_{2}=0.075, \gamma_{3}=0.188$, respectively. The coefficient $\gamma$, inversely proportional to the heat conductivity factor, increases on cooling the sample. An insignificant difference between the experimental results and Eq. (3) for low mirowave power is caused probably by the presence of a power threshold for fluxon nucleation into the weak contact.

\section{Discussion}

After the discovery of high-temperature superconductivity many authors have confirmed the presence of internal Josephson junctions in the relevant materials. The application of the classical description of macroscopic Josephson junctions to the phenomena taking place in ceramic high-temperature superconductors proved to be particularly attractive.

According to the Josephson junction $I(U)$ characteristics, the fluctuating voltage $V$ appears when the current exceeds its critical value, as:

$$
V=R\left[I^{2}-I_{\mathrm{c}}^{2}\right]^{1 / 2},
$$

where $R$ denotes the resistance of the Josephson junction in the normal state. The critical current $I_{c}$ depends on the magnetic field according to the relation:

$$
I_{\mathrm{c}}=I_{\mathrm{c}}(0)\left|\frac{\sin \left(\pi \phi / \phi_{0}\right)}{\pi \phi / \phi_{0}}\right|,
$$

where $I_{\mathrm{c}}(0)$ is the critical current, in zero magnetic field. When $I>I_{\mathrm{c}}$, the fluctuating voltage will be described by the following formula:

$$
V=R I_{\mathrm{c}}(0)\left[1-\left(\frac{\sin \left(\pi \phi / \phi_{0}\right)}{\pi \phi / \phi_{0}}\right)^{2}\right]^{1 / 2}
$$

This voltage generates dissipation and the dissipated power related to the fluctuating current loss in the junction equals $P=V^{2} / R$.

In the granular high-temperature superconductors we consider a system of internal Josephson junctions formed among the grains. Thus, applying EPR methodology, the microwa ve absorption signal, proportional to the microwave power loss in the interaction with the system of internal Josephson junctions, can be described by the following expression:

$$
P(0)=R I_{\mathrm{c}}^{2}(0)\left[1-\left(\frac{\sin \left(\pi \phi / \phi_{0}\right.}{\pi \phi / \phi_{0}}\right)^{2}\right] .
$$

The interactions between the microwaves, the JJS and the bulk sample compete to reach a thermodynamical equilibrium and only then we can define a local temperature of the junction [8]. 
Assuming the temperature dependence of the critical current to be similar to that of the critical field (Eq. (3)), the influence of the microwaves on the critical current can be described as:

$$
I_{\mathrm{c}}\left(T, P_{\text {micr }}\right)=I_{\mathrm{c}}(0,0)\left[1-\left(\frac{T+\gamma P_{\text {micr }}}{T_{\mathrm{c}}}\right)^{2}\right] \text {, }
$$

where $\gamma=a / K^{\prime}$ from Eq. (2), and $P_{\text {micr }}$ is the microwave power irradiating the junction.

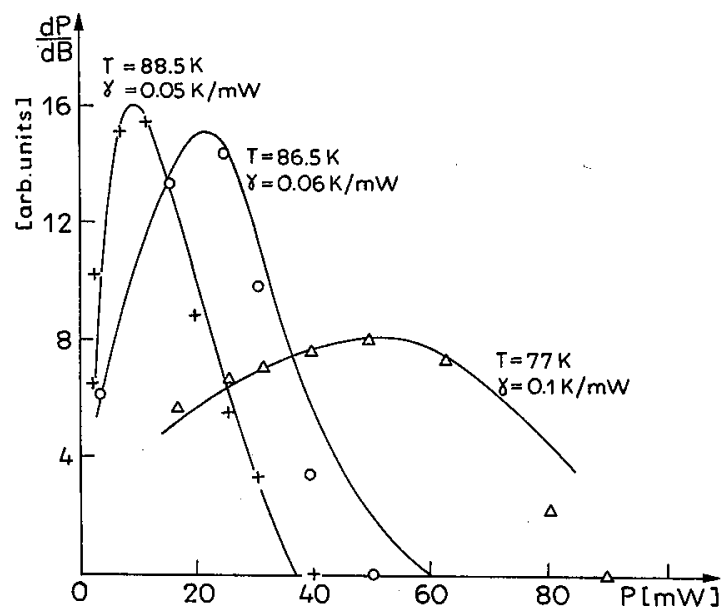

Fig. 3. Amplitude of JMA signal $(\mathrm{d} P / \mathrm{d} B)$ vs. microwave power for different temperatures. Solid lines represent experimental approximation by Eq. (11).

Let us assume that the amplitude of the JMA signal is proportional to $P_{\text {micr }} / P_{0}$, where $P_{\text {micr }}$ is the applied microwave power and $P_{0}$ is the maximum power absorbed by the system:

$$
P_{\mathrm{JMA}}=P\left(T, P_{\text {micr }}, \phi\right) P_{\text {micr }} / P_{0},
$$

where $P_{0}=R I_{\mathrm{c}}^{2}(0,0)$. Thus, the dependence of the JMA line shape on the microwave effect, when JJS is overheated $\left(T^{*}>T\right)$, can be written as:

$$
P_{\mathrm{JMA}}=P_{\text {micr }}\left[1-\left(\frac{T+\gamma P_{\text {micr }}}{T_{\mathrm{c}}}\right)^{2}\right]^{2}\left[1-\left(\frac{\sin \left(\pi \phi / \phi_{0}\right)}{\pi \phi / \phi_{0}}\right)^{2}\right] .
$$

Eq. (10) is the most general formula describing JMA signal. The first part describes the amplitude of the JMA vs. microwave power $P_{\text {micr }}$, while the second part of Eq. (10) shows the dependence of JMA signal on the magnetic field. The expression describing the temperature dependence of the amplitude of the JMA signal is following:

$$
P_{\mathrm{JMA}}\left(T, P_{\text {micr }}\right)=P_{\text {micr }}\left[1-\left(\frac{T+\gamma P_{\text {micr }}}{T_{\mathrm{c}}}\right)^{2}\right]^{2} .
$$



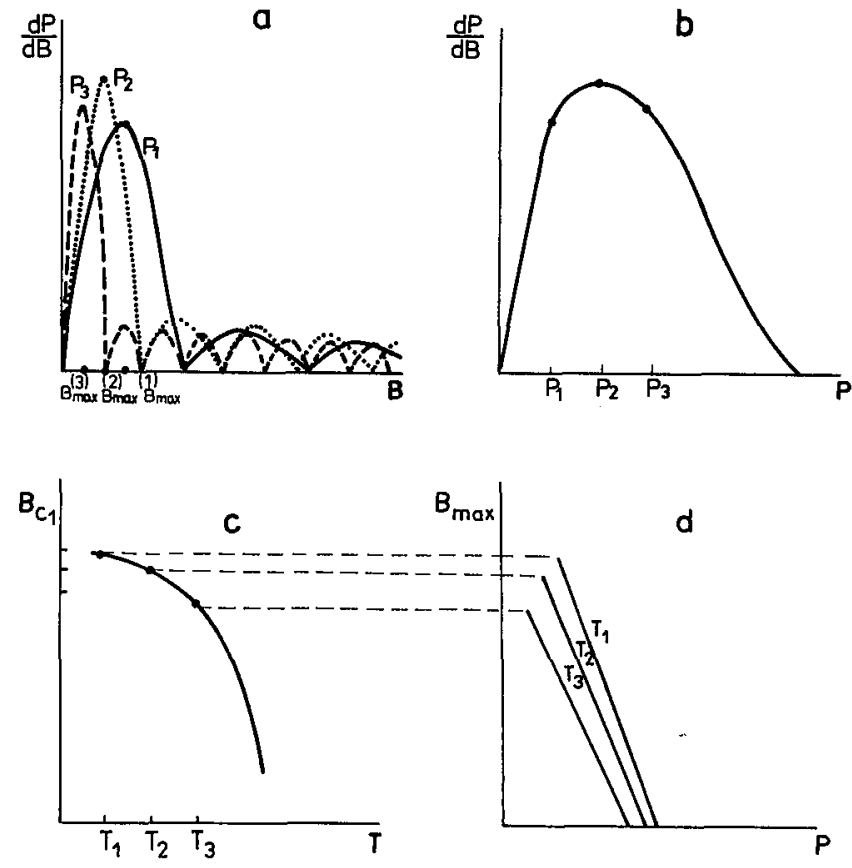

Fig. 4. A schematic presentation of JMA line-shape changes for different microwave power $P_{1}<P_{2}<P_{3}(\mathrm{a})$, the signal amplitude and $B_{\max }$ vs. microwave power (b, d) and extrapolated (see text) $B_{c 1}$ versus temperature (c), plotted according to Eq. (10).

The function (11) illustrates the behaviour of the JMA amplitude under the effect of microwaves, and is also in good agreement with the results shown in Fig. 3, where the JMA signal amplitude increases, achieves the maximum and then decreases with increasing microwave power.

The effect of microwave power on the JMA line shape, the signal amplitude and $B_{\max }$ field are illustrated schematically in Fig. 4(a). The JMA amplitude changes due to the applied microwave power are presented in Fig. 4(b). Fig. 4(d) illustrates the effect of microwave power on the maximum of the microwave absorption signal $B_{\max }$, which can be described by Eq. (10) assuming the presence of a microwave power threshold for fluxon nucleation into the weak contact. Figure 4 (c) presents the temperature dependence of the critical field $B_{\mathrm{c1}}$ whose value has been extrapolated to zero microwave power from the experimental dependences shown in Fig. 2.

The disappearence of the JMA signal observed for high microwave power in spite of the bulk sample temperature $T<T_{c}$, is due to the overheating of JJS up to $T^{*}>T_{\mathbf{c}}$, as in the experiments reported in [8].

The investigation of the JMA signal gives the opportunity to analyse the interaction of JJS with the microwaves and bulk granular superconducting sample 
in the terms of thermodynamics of a system which contains three different reservoirs; the energy flows from the reservoir of microwaves to bulk sample through the JJS.

\section{References}

[1] J.G. Bednorz, K.A. Mueller, Z. Phys. B, Condens. Matter 64, 189 (1986).

[2] J. Stankowski, P.K. Kahol, N.S Dalal, J.S. Moodera, Phys. Rev. B, Condens. Matter 36, 7126 (1987).

[3] A. Dulcic, B. Leontic, M. Peric, B. Rakvin, Europhys. Lett. 4, 1403 (1987).

[4] A.M. Portis, K.W. Blazey, K.A. Mueller, J.G. Bednorz, Europhys. Lett. 5, 467 (1988).

[5] K.W. Blazey, A.M. Portis, J.G. Bednorz, Solid State Commun. 65, 1153 (1988).

[6] A. Dulcic, R.H. Crepau, J.H. Freed, in: Magnetic Resonance and Related Phenomena, Proc. 24th Ampere Congress, Poznań 1988, ed. J. Stankowski, Elsevier, Amsterdam 1989, p. 215.

[7] J. Stankowski, J. Pichet, B. Czyżak, in: Proc. Int. Workshop on Magnetic Resonance, ed. N. Yordanov, Scientific World Pub., Singapore 1989, p. 271.

[8] J. Stankowski, B. Czyżak, J. Martinek, Phys. Rev. B, Condens. Matter 42, 10243 (1990).

[9] J. Stankowski, B. Czyżak, Z. Trybuła, J.E. Drumheller, submitted to J. Phys., Condens. Matter.

[10] A. Barone, G. Paterno, Physics and Applications of the Josephson Effect, Wiley, New York 1982.

[11] Y. Maniwa, A. Grupp, F. Hentsch, M. Mehring, Physica C 156, 755 (1988).

[12] J. Stankowski, B. Czyżak, M. Krupski, J. Baszyński, T. Datta, C. Almasan, Z.Z. Sheng, A.M. Hermann, Physica C 160, 170 (1988).

[13] Z. Trybuła, J.E. Drumheller, J. Stankowski, in: 34th Annual Conf. on Magnetism and Magnetic Materials, Boston 1989, p.143.

[14] J.E. Drumheller, Z. Trybula, J. Stankowski, J. Appl. Phys. 67, 5041 (1990).

[15] J. Stankowski, J. Pichet, C.P. Poole, T. Datta, P.K. Kahol, N.S. Dalal, J.S. Moodera, J.E. Drumheller, S.L. Hutton, R.S. Rubins, Ferroelectrics 78, 231 (1988).

[16] B. Czyżak, J. Stankowski, Phys. Status Solidi B 166, 219 (1991).

[17] B. Czyżak, T. Żuk, J. Stankowski, Acta Phys. Pol. A78, 769 (1990). 